\title{
Minimal Waiting Time Assignment of Subcarriers and Power for OFDMA System
}

\author{
Xiang YANG \\ Dept. of Electron. and Info, The South Chinese University \\ of Technology, \\ Wushan, Guangzhou, P.R. China. \\ eexyang@126.com
}

\begin{abstract}
This paper presents a new method for subcarriers and power allocation for Orthogonal Frequency Division Multiple Access (OFDMA) with the purpose of minimizing the instantaneous buffering latency of all users. Numerical results show that the average packet delay can be reduced by up to $50 \%$ and the spectrum utilization can be increased by $0.5 \mathrm{bits} / \mathrm{s} / \mathrm{Hz}$ when compared to the scheme used in IEEE802.16a.
\end{abstract}

Keywords - OFDMA, waiting time, adaptive resource allocation, quality of service (QoS)

\section{INTRODUCTION}

OFDMA has been adopted by IEEE802.16a as an option [1]. It allows multiple users to share a physical channel while providing resistance to Inter-Symbol Interference (ISI) and frequency selective fading [2]. Methods for enhancing system performance in the use of OFDMA include subcarriers allocation, bit loading and power control. In single user environment, the use of water-pouring scheme for bit loading and power control in OFDM can optimize the spectrum utilization [3]. In multi-user environment, the subcarriers are shared by multiple users and so that is necessary when a subcarrier suitable for a user may also be suitable for another user who may not have other good subcarriers to use. Given the users traffic, some techniques were proposed to minimize the total transmission power in OFDM [5-7]. For OFDMA, techniques were proposed which minimize the total transmission power while guaranteeing the fairness among users [4] and maximizing the spectrum utilization[8].

These approaches assume all users have sufficient traffic or the transmit queues are always full. We propose a new minimal waiting time assignment scheme without this restriction and aims at minimizing the average delay of users in each scheduling period.

\section{SYSTEM MODEL}

\section{A. OFDMA System Model}

Fig. 1 shows an OFDMA system at a base station (BS). The multiple data streams from users are assigned channel resources by the Adaptive Resource Allocation (ARA) Control Module. It allocates a set of OFDMA subcarriers for each of the $\mathrm{K}$ users and determines the data rate of each of the $\mathrm{N}$ subcarriers.

\author{
Tak-Shing Peter YUM \\ Dept. of Info. Eng., The Chinese University of Hong Kong, \\ Shatin, Hong Kong. \\ yum@ie.cuhk.edu.hk
}

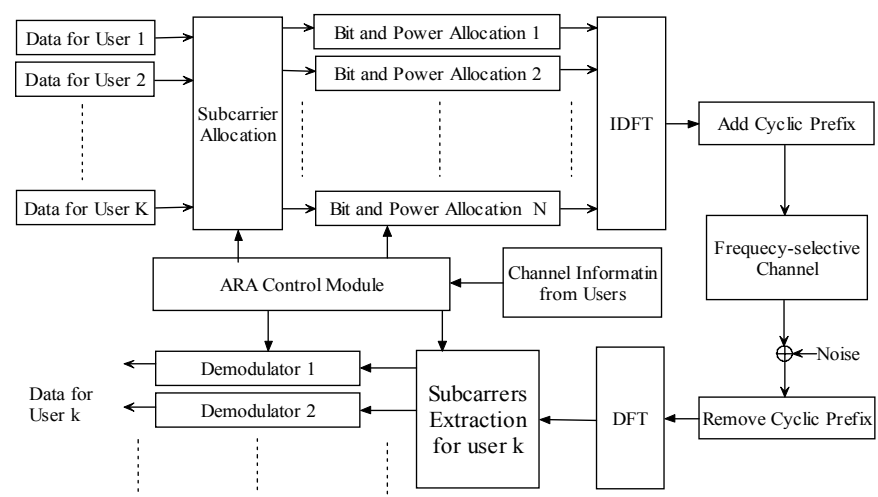

Figure 1. OFDMA with Adaptive Resource Allocation

OFDMA converts a wideband frequency selective fading channel into a set of narrowband frequency flat fading channels (called subcarriers here) by Inverse Discrete Fourier Transform (IDFT). To alleviate intersymbol interference, a guard time is inserted between adjacent frames. This guard time is called cyclic prefix and it needs to be larger than the channel propagation delay. If $\mathrm{Td}$ is the sampling interval of the system and $\eta$ is the length of cyclic prefix, the duration of one OFDMA frame is $T_{f}=(N+\eta) T_{d}$. The modulated prefixappended IDFT signal is then sent through the frequency selective fading channel.

At the receiving side of the user $k$, the cyclic prefix is first removed after demodulation. Then the whole OFDMA frame is transformed into frequency domain by DFT. The subcarriers associated with user $k$ are extracted and demodulated. The resulting data streams are then combined before passing to the upper layer of user $k$.

\section{B. Bit Loading and Power Control}

We assume the base station has perfect knowledge of the channel gain matrix $G=\left\lfloor g_{k, n}\right\rfloor$ where $g_{k, n}$ is the channel gain of subcarrier $n$ of user $k$ and is assumed constant over each resource allocation interval. Let $b_{k, n}$ and $p_{k, n}$ be the bit loading and the transmission power of subcarrier $n$ of user $k$ respectively. Let $v\left(b_{k, n}\right)$ be the required received power with unity channel gain for reliable reception of $b_{k, n}$ bits per 
symbol under a specified $B E R_{k}^{*}$ for subcarrier $n$ of user $k$. The SNR of the received signal can be expressed as

$$
\operatorname{SNR}\left(b_{k, n}\right)=\frac{v\left(b_{k, n}\right)}{\delta^{2}}
$$

The required received power $v\left(b_{k, n}\right)$ was derived in [9] as

$$
v\left(b_{k, n}\right)=p_{k, n} g_{k, n}=\frac{\left[Q^{-1}\left(B E R_{k}^{*} / 4\right)\right]^{2}\left(2^{b_{k, n}}-1\right) \delta^{2}}{3},
$$

where $Q^{-1}(\bullet)$ is the inverse complementary error function and $\delta^{2}$ is the noise power. Solving for $b_{k, n}$, we obtain

$$
b_{k, n}=\log _{2}\left(\frac{3 p_{k, n} g_{k, n}}{\left[Q^{-1}\left(B E R_{k}^{*} / 4\right)\right]^{2} \delta^{2}}+1\right)=\log _{2}\left(A_{k} p_{k, n} g_{k, n}+1\right) \text {, }
$$

where $A_{k}=\frac{3}{\left[Q^{-1}\left(B E R_{k}^{*} / 4\right)\right]^{2} \delta^{2}}$.

\section{THE MINIMAL WAITING TIME OPTIMIZATION}

We assume data packets for different users are organized in queues, one for each user. The packet scheduler assigns these packets to the $\mathrm{N}$ subcarriers in the physical layer for transmission.

The BS schedules transmissions in intervals of $T_{s}$ (a multiple of $T_{f}$.) OFDMA assigns each subcarrier to only one user and we express this assignment as

$$
\sigma_{k, n}=\left\{\begin{array}{ll}
1, & \text { if subcarrierisallocatedbuserk } \\
0, & \text { otherwise }
\end{array} .\right.
$$

The total data rate $r_{k}$ for user $k$ is therefore

$$
r_{k}=\sum_{n=1}^{N} \frac{\sigma_{k, n} b_{k, n}}{T_{f}}
$$

Let $Q_{k}$ be the instantaneous buffer backlog (in bits) of user $k$ at the beginning of the scheduling period. Then, the time $W_{k}$ needed to clear this backlog data rate $r_{k}$ is

$$
W_{k}=Q_{k} / r_{k}=\frac{Q_{k}}{\sum_{n=1}^{N} \frac{\sigma_{k, n} b_{k, n}}{T_{f}}}=\frac{T_{f} Q_{k}}{\sum_{n=1}^{N} \sigma_{k, n} \log _{2}\left(A_{k} p_{k, n} g_{k, n}+1\right)}
$$

Let $W=\sum_{k=1}^{K} W_{k}$ be the total buffer backlog. Our proposed minimal waiting time optimization problem is to assign carriers and power so as reduce $W$ as much as possible each scheduling interval and is formulated as follows:

Minimizing $W$, w.r.t $\left\{p_{k, n}\right\},\left\{\sigma_{k, n}\right\}$.

Subject to

$$
\sum_{k=1}^{K} \sum_{n=1}^{N} \sigma_{k, n} p_{k, n} \leq P_{\text {Max }}, \quad \text { and } \sum_{k=1}^{K} \sigma_{k, n} \leq 1, \forall n \in\{1, \ldots N\} \text {, }
$$

where $P_{M a x}$ is the total available transmission power in the base station. This is a mixed integer programming problem. We therefore propose the following heuristic for a sub-optimal solution.

\section{HEURISTIC SOLUTION}

The methods proposed for adaptive resource allocation in [5-8] are based on the greedy approach. With that, users with poor channel conditions are sometimes sacrificed in favor of users with good channel conditions. The method proposed in [4] balances the fairness in data transmit rates among users, but it ignores the non-uniform bandwidth demand of users in the link layer. Addressing these problems, we propose the following four-parts algorithm, from A to D.

\section{A. Subcarrier Allocation}

This is the initialization part of the algorithm. Without knowledge from the physical layer, it is natural to allocate subcarriers proportional to the queue lengths. This allocation will be adjusted through iteration in part $\mathrm{D}$ when channel condition information is used.

INPUT : queue length $\left\{Q_{k}\right\}$.

OUPUT : $\left\{u_{k}\right\}$ where $u_{k}$ is the number of subcarriers allocated to user $k$.

1. For $k=1, \ldots K, C_{k}=\phi$.

2. For $k=1, \ldots K, u_{k}=\left\lfloor\frac{Q_{k}}{\sum_{k=1}^{K} Q_{k}} N\right\rfloor$ and $y_{k}=\frac{Q_{k}}{\sum_{k=1}^{K} Q_{k}} N-u_{k}$. ( $u_{k}$ and $y_{k}$ are the integer and fractional parts of the number of assigned subcarriers.)

3. Do until $\sum_{k=1}^{K} u_{k}=N$.

$3.1 k^{*}=\underset{1 \leq k \leq K}{\arg \max }\left\{y_{k}\right\}$,

(User $k^{*}$ is identified to have the maximum amount of unsatisfied bandwidth demand.)

$3.2 u_{k^{*}}=u_{k^{*}}+1$,

(User $k^{*}$ is assigned one more subcarrier.)

$3.3 y_{k^{*}}=0$.

\section{END}




\section{B. Subcarrier Assignment}

This part is on the assignment of specific subcarriers according to

- The allocated number of subcarriers;

- The subcarrier channel conditions, for optimizing spectrum utilization [6].

INPUT : $\left\{u_{k}\right\}$ and channel gain matrix G.

OUPUT : subcarrier assignments $\left\{\mathbf{C}_{k}\right\}$ where $\mathbf{C}_{k}$ is the set of subcarriers for user $k$.

1. For all $k$ and $n, h_{k, n}=g_{k, n}$,

2. $\left\{k^{*}, n^{*}\right\} \leftarrow \underset{1 \leq k \leq K, 1 \leq n \leq N}{\operatorname{argmax}}\left\{h_{k, n}\right\}, h_{k^{*}, n^{*}}=\underset{1 \leq k \leq K, 1 \leq n \leq N}{\arg \max }\left\{h_{k, n}\right\}$.

3. If $\left|\mathbf{C}_{k^{*}}\right|<u_{k^{*}}$,

$$
\mathbf{C}_{k^{*}}=\mathbf{C}_{k^{*}} \cup\left\{n^{*}\right\}, h_{k, n^{*}}=0, k=1, \ldots K .
$$

else, $h_{k^{*}, n}=0$ for all $n$.

4. If $\sum_{k=1}^{K}\left|\mathbf{C}_{k}\right|<N$, go to 2 .

5. END

\section{Bit and Power Allocation}

In this part of the algorithm, we present a procedure for incrementing the bit loading of the assigned subcarriers (or incrementing subcarriers capacity) to achieve the optimization object until the total power constraint is reached. Let $\Delta p_{k, n}$ be the minimum power increment needed for increasing bit loading by 1 for subcarrier $n$ of user $k$.

INPUT: subcarrier assignments $\left\{\mathbf{C}_{k}\right\}$ and channel gain matrix $\mathrm{G}$

OUPUT: power allocation $p_{k, n}$ and bit loading $b_{k, n}$ for every subcarrier.

1. $p_{k, n}=0$ and $b_{k, n}=0$ for all $n$ and all $k$.

2. For all $k$,

$n^{*}=\underset{n \in C_{i}}{\arg \max }\left\{g_{k, n}\right\}, b_{k, n^{*}}=1$ and $p_{k, n^{*}}=\frac{S N R\left(b_{k, n^{*}}\right) \delta^{2}}{g_{k, n^{*}}}$.

( $\operatorname{SNR}\left(b_{k, n^{*}}\right)$ is the required receiver SNR corresponding to bit loading $b_{k, n^{*}} ; p_{k, n^{*}}$ is the required power under channel gain $g_{k, n^{*}} \cdot$ )

3. For all $k, b_{k}=\sum_{n \in C_{i}} b_{k, n}$.
( $b_{k}$ is the total bits for user $k$ )

4. While $\sum_{k=1}^{K} \sum_{n \in C} p_{k, n}<P_{\operatorname{Max}}$, do

$4.1 P_{L}=P_{M a x}-\sum_{k=1}^{K} \sum_{n \in \mathbf{C}_{k}} p_{k, n}$.

( $P_{L}$ is the total residual power. )

4.2 For all $k$,

$$
\begin{aligned}
\Delta p_{k, n} & =\left\{\operatorname{SNR}\left(b_{k, n}+1\right) \delta^{2}-\operatorname{SNR}\left(b_{k, n}\right) \delta^{2}\right\} / g_{k, n}, \quad n \in C_{k} \\
n^{*} & =\underset{n \in C_{k}, \Delta p_{k, n}<P_{L}}{\arg \min } \Delta p_{k, n} .
\end{aligned}
$$

(For each user $k$, we find subcarrier $n^{*}$ that requires the smallest power increment for increasing bit loading by 1 . That power increment must be less than the residual power, or the total power would exceed $P_{\operatorname{Max}}$ otherwise. )

$$
4.3 k^{*}=\underset{1 \leq k \leq K}{\arg \max } \frac{\Delta W_{k}}{\Delta p_{k, n^{\prime}}}=\frac{W_{k}\left(b_{k}\right)-W_{k}\left(b_{k}+1\right)}{\Delta p_{k, n^{\prime}}} .
$$

( Find user $k^{*}$ which gets the largest waiting time reduction per unit power increment. )

$$
\begin{aligned}
& 4.4 p_{k^{*}, n^{*}}=p_{k^{*}, n^{*}}+\Delta p_{k^{*}, n^{*}}, b_{k^{*}, n^{*}}=b_{k^{*}, n^{*}}+1 . \\
& 4.5 b_{k^{*}}=b_{k^{*}}+1 .
\end{aligned}
$$

\section{END.}

In summary, in part $\mathrm{C}$ bits on the subcarriers are incremented step by step for users that can get the most waiting time reduction per unit power increase.

\section{Subcarriers Allocation Adjustment}

Recall that in Part A the number of subcarriers is assigned without regard to channel quality. In this part we make adjustments by reassigning a subcarrier from the most resource-rich user to the least resource-rich user. Let $p_{k}$ be the total power allocated to user $k$. Then a small $W_{k} / p_{k}$ value indicates resource-richness and vice versa. To bound the computation time, the number of iterations $\gamma$ is bounded to $\gamma_{\max }$.

1. $\gamma=0$, While $\frac{W^{(\text {old })}-W^{(\text {new })}}{W^{(\text {old })}}$ and $\gamma<\gamma_{\max }$ do

$1.1 k_{\max }=\underset{1 \leq k \leq K}{\arg \max } \frac{W_{k}}{p_{k}}$ and $k_{\min }=\underset{1 \leq k \leq K}{\arg \min } \frac{W_{k}}{p_{k}}$.

$1.2 u_{k_{\max }}=u_{k_{\max }}+1$ and $u_{k_{\min }}=u_{k_{\min }}-1$.

1.3 Repeat Part B.

1.4 Repeat Part C. 
$1.5 \gamma=\gamma+1$.

\section{END.}

In summary, Part A is the initialization phase. Parts B, C and $\mathrm{D}$ form a loop of successive relaxation for subcarriers allocations. This is a standard technique in optimization for the iterative solution of multiple sets of variables.

\section{NUMERICAL RESULTS}

For the simulation scheme of the optimal multiplexing algorithm, we choose a $5 \mathrm{MHz}$ channel with a total 128 subcarriers. Let there be $k=10$ users sharing these subcarriers. The cyclic prefix length is set at $\eta=20$ symbols. These and a few other channel parameters are summarized in Table I. The bit loadings, modulation schemes, coding rates and the required receiver SNR used in our simulation are stated in Table II. These parameters are adopted from IEEE 802.16a [1]. Rayleigh fading channel with 3 symbol-spaced taps and an exponential decaying profile is assumed also in [10-11]. We use two cases to compare the proposed minimal waiting time assignment with the Fixed-assignment used in IEEE 802.16a [1] and the Fair assignment proposed in [4].

\section{TABLE I. SIMULATION PARAMETERS}

\begin{tabular}{|c|c|}
\hline Bandwidth & $\mathbf{5 M H z}$ \\
\hline Number of Subcarriers N & $\mathbf{1 2 8}$ \\
\hline Number of Users K & $\mathbf{1 0}$ \\
\hline Cyclic Prefix Length $\eta$ & $\mathbf{2 0 ~ s y m b o l s}$ \\
\hline OFDMA symbol duration $T_{f}$ & $\mathbf{2 9 . 6} \mu \mathrm{s}$ \\
\hline Resource Allocation Interval $T_{s}$ & $\mathbf{5 9 2} \mu \mathrm{s}$ \\
\hline Noise Power & $\mathbf{- 5 ~ d B m W}$ \\
\hline BER Requirement & $10^{-6}$ \\
\hline
\end{tabular}

TABLE II. TRANSMISSION MODES IN IEEE 802.16A

\begin{tabular}{|c|c|c|c|c|}
\hline $\begin{array}{c}\text { Rate } \\
\text { ID }\end{array}$ & $\begin{array}{c}\text { Bits Loading in } \\
\text { Subcarrier }\end{array}$ & Modulation & Coding Rate & $\begin{array}{c}\text { Receiver SNR } \\
(\mathrm{dB})\end{array}$ \\
\hline R0 & 1 & QPSK & $1 / 2$ & 9.4 \\
\hline R2 & 2 & 16-QAM & $1 / 2$ & 16.4 \\
\hline R3 & 3 & 16-QAM & $3 / 4$ & 18.2 \\
\hline R4 & 4 & 64-QAM & $2 / 3$ & 22.7 \\
\hline
\end{tabular}

The Fixed assignment operates in two steps. Step 1 allocates subcarriers to users without considering the channel condition. Step2 performs bit-loading (i.e. adaptive modulation) according to channel conditions. Fair assignment allocates subcarriers and performs bit-loading to equalize the date rates of all users. We compare these methods in the following two cases:

\section{Case 1: CBR Traffic}

In Fig. 2 we show the data loss probability as a function of the total data rate for the three assignment methods. Let all users have the same fixed data rate (CBR) and at the total transmission power be $44 \mathrm{dBmW}$. We set $W_{k}=1 s$ as the threshold for dropping data. Fig.3 shows the same for non- uniform data rates where proportion of traffic for the 10 users are as follows: $(0.170,0.084,0.055,0.086,0.087,0.083,0.209$, $0.105,0.056,0.065)$. Both figures show that the minimal waiting time assignment can give significant reduction of loss probabilities.

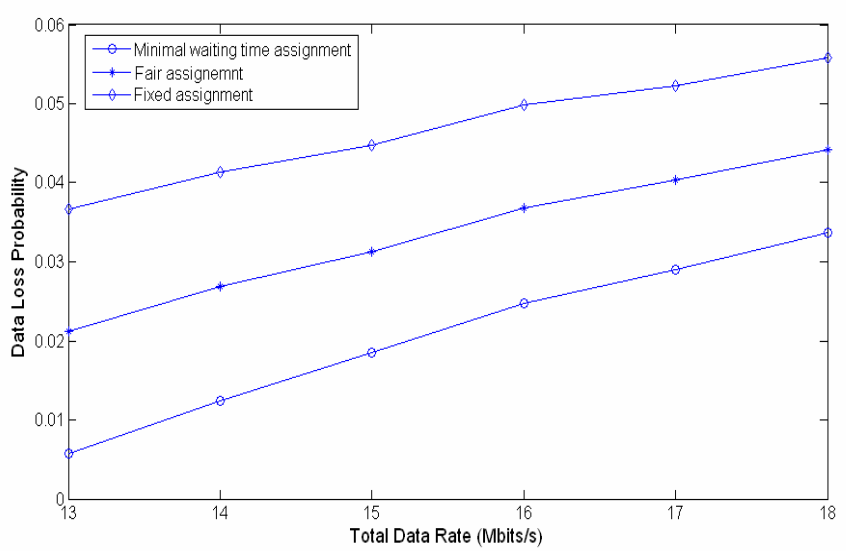

Figure 2. Packet Loss Probability (uniform traffic)

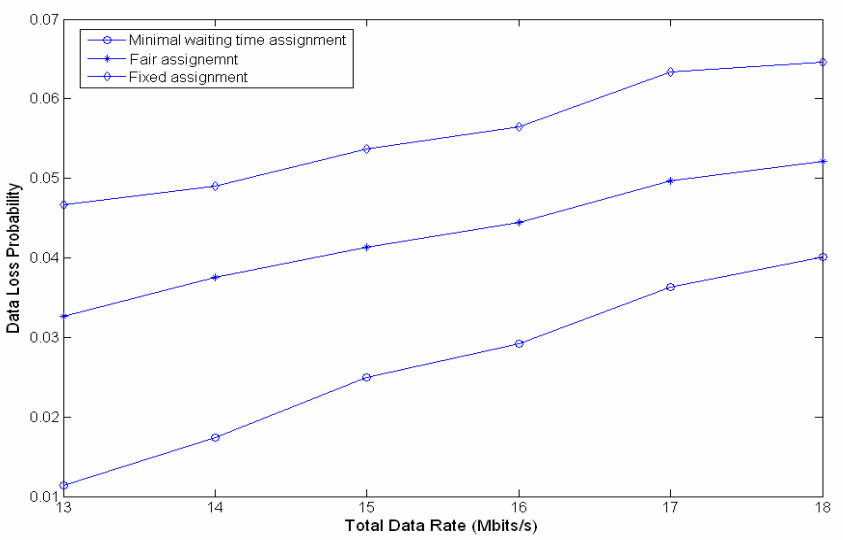

Figure 3. Packet Loss Probability (non-uniform traffic)

\section{Case 2: Bursty Traffic}

We now study the performance of bursty traffic in wireless Internet application. We assume the overall arrival of packets to the base-station is a Poisson process with rate $\lambda$ packets per scheduling interval. The packet length distribution follows that in [12-13] with a mean of 364.7 bytes. The detail distribution is 64 bytes $(41.5 \%), 1518$ bytes $(8.2 \%), 558$ bytes $(7.0 \%), 90$ bytes $(5.9 \%)$, and 570 bytes $(5.5 \%)$. Each packet is equally likely to join the 10 queues of the 10 users.

Fig.4 shows the average packet delay as a function of power budget $p_{\operatorname{Max}}$ with $\lambda=21$. It is seen that minimal waiting time assignment gives significantly smaller average packet delay than the other two schemes. This advantage is a result of exploiting the dynamic carrier assignment and the preferential treatment to users with longer queue lengths. 
Fig. 5 shows the average packet delay as a function of $\lambda$, with $P_{M a x}=44 d B m W$. It is seen that the minimal waiting time assignment can reduce the packet delay over the entire traffic range shown.

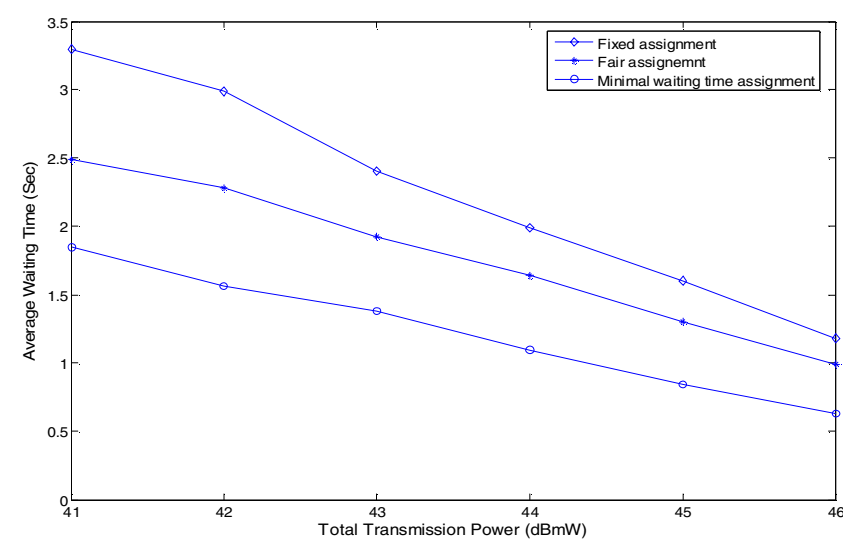

Figure 4. The Average Packet delay as a Function of $P_{\text {Max }}, \lambda=21$

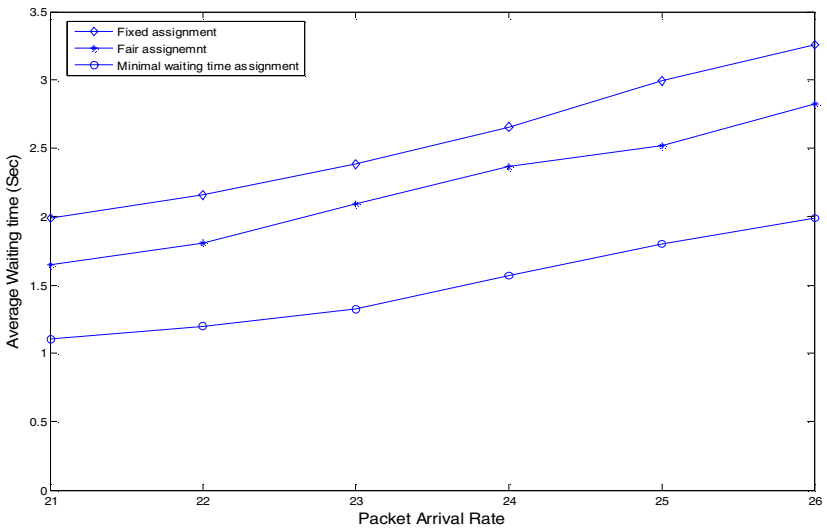

Figure 5 The Average Packet Delay as a Function of $\lambda$

Fig.6 compares the spectrum utilization of the three assignment schemes as a Function of $P_{M a x}$. It shows that when $P_{M a x}>43 \mathrm{dBmW}$, the minimal waiting time assignment can increase the spectrum utilization by more than $0.5 \mathrm{bit} / \mathrm{s} / \mathrm{Hz}$ when compared to Fixed-assignment. The capacity increase at $P_{\text {Max }}=45 \mathrm{dBmW}$ is about $30 \%$.

\section{CONCLUSION}

In this paper, a new approach for assigning subcarriers and power for OFDMA is proposed. Extensive computer simulation shows that using the overall waiting time for the optimization objective can significantly improve the throughdelay performance of both Internet traffic and CBR traffic over diverse operating conditions.

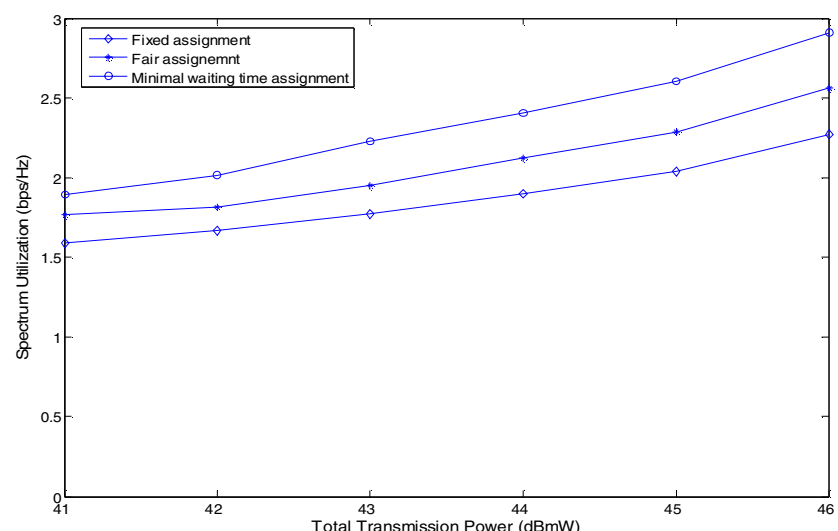

Figure 6. The Spectrum Utilization as a Function of $P_{\operatorname{Max}}$

\section{REFERENCE}

[1] "Air Interface for fixed Broadband Wireless Access SystemsAmendment 2: Medium Access Control Modifications and Additional Physical Layer Specifications for 2-11GHz", IEEE Std 802.16a-2003.

[2] Justin Chuang and Nelson Sollenberger, "Beyond 3G: wideband wireless data access based on OFDM and dynamic packet assignment", IEEE Commun. Magazine, vol. 38, pp.78- 87, July. 2000.

[3] Guocong Song and $\mathrm{Ye} \mathrm{Li,} \mathrm{"Adaptive} \mathrm{resource} \mathrm{allocation} \mathrm{based} \mathrm{on} \mathrm{utility}$ optimization in OFDM", IEEE GLOBECOM '03, vol. 2, pp.586-590. Dec. 2003

[4] Mustafa Ergen, Sinem Coleri, and Parvin Varaiya, "QoS aware adaptive resource allocation techniques for fair scheduling in OFDMA base Broadband wireless access systems.” IEEE Trans. Broadcasting, vol. 49, no. 4 , pp.362-370, Dec. 2003.

[5] C. Y. Wong, R. S. Cheng, K. B. Letaief and R. D. Murch, "Multiuser OFDM with adaptive subcarrier, bit, and power allocation," IEEE J. Select. reas Commun., vol. 17, no.10, pp. 1747-1758., Oct. 1999.

[6] Teo Choon Heng Alen, A. S. Madhukumar and Francois Chin, "Capacity enhancement of a multi-user OFDM system using dynamic frequency allocation", IEEE Trans. Broadcasting, vol.49, pp. A 344-353, Dec. 2003

[7] Jiho Jang and Kwang Bok Lee, "Transmit power adaptation for multiuser OFDM systems", IEEE Journal on Selected Area in Commun., vol.21, pp. 171-178, Feb. 2003.

[8] Didem kivanc, Guoqing Li, and Hui Liu, "Computationally efficient Bandwidth allocation and power control for OFDMA", IEEE Trans.Wireless Commun., vol.2, pp.1150-1158, Nov. 2003.

[9] J. G. Proakis, Digital communication, McGraw Hill, 2001.

[10] IEEE C80216e-03 03, OFDMA System Simulation in a Single/Multi Cell Configuration.

[11] Adrian Bohdanowicz, Gerard J.M. Janssen and Slawomir Pietrzyk, “ Wideband indoor and outdoor multipath channel measurements at 17GHz,” in Proc. IEEE VTC' 99, Sep. 1999, vol. 4, pp. 1998-2003.

[12] Victor. S. Frost and Benjamin. Melamed, "Traffic modeling for telecommunications networks," IEEE commun. Mag., vol. 32, pp. 7081, Mar. 1994.

[13] Kenji. Yoshigoe and Kenneth J. Christensen, "An evolution to crossbar switches with virtual output queuing and buffered cross points", IEEE Trans. Network., vol.17, pp.48-56, Sept. 2003. 